\title{
Moderate Conventionalism and Cultural Appropriation
}

\author{
Juha Räikkä* \& Mikko M. Puumala** \\ *University of Turku, Åbo, Finland, jraikka@utu.fi \\ ${ }^{*}$ University of Turku, Åbo, Finland, mikko.puumala@utu.fi \\ DOI: http://dx.doi.org/10.5324/eip.v13i1.2876
}

(cc) BY
Attribution 4.0 International License, which permits unrestricted use, distribution, and
reproduction in any medium, provided the original author and source are credited.

Cultural appropriation, also called cultural borrowing, has been the topic of much discussion in recent years. Roughly speaking, cultural appropriation happens when someone outside of a cultural or ethnic group takes or uses some object that is characteristic or in some way important to the group without the group's permission. Individuals who find cultural appropriation (or borrowing) unproblematic have often argued that if we express moral criticism of the use of traditional Sami outfits by non-Sami, then we are logically committed to criticize all kinds of habits that are clearly acceptable -such as using jeans, eating pizza or drinking tea. However, we will argue that in many cases that objection is problematic. We point out that if one social habit or practice is prohibited (or supported) by existing social conventions but another is not, then there is a convention difference between the cases. The convention difference is in turn a morally relevant difference, or so we aim to show. We refer to "moderate conventionalism," according to which existing social conventions are morally relevant facts that should be taken into account when choosing how to act, whatever the content of the conventions happens to be. The claim is analogous with the traditional view that laws have some moral relevance and binding force independent of their content.

Keywords: cultural appropriation, conventionalism, moderate conventionalism, convention difference

\section{Introduction}

Cultural appropriation, also called cultural borrowing, has been the topic of much discussion in recent years. Roughly speaking, cultural appropriation happens when someone outside a cultural or an ethnic group takes or uses some object that is characteristic or in some way important to the group without the group's permission. A deeper understanding of cultural appropriation would require an historical description of the phenomenon, but here we can shed some light on the issue by means of concrete examples. A famous example of a debate that has centered around the notion of cultural appropriation concerns the question of whether it is morally appropriate for white men to play the blues (see Rudinow 1994; Young 1994). Another example of such a debate is a recent discussion about 
outsiders' alleged right to wear traditional clothing of indigenous groups (see Lynskey 2014). Is it morally acceptable to wear headgear of a Native American indigenous group to a costume party? In the former discussion the focus has been on the right to produce certain goods, but in the latter the focus has shifted to the issue of whether outsiders have a moral right to use the goods of some cultural groups at all. The examples are different, and they correctly suggest that cultural appropriation can come in many forms. Notice that the debate on immaterial property rights concerns many issues that are related to the cultural borrowing. Music, cuisine, words, and medicine are typical examples (for a discussion, see Ziff \& Rao 1997; Young \& Brunk 2009).

Is cultural appropriation morally problematic? If it is, we should ask when it is problematic and why it is problematic. The common arguments against cultural appropriation vary greatly. It has been argued that cultural appropriation is theft, especially when a cultural group that claims to have created something receives no compensation (see Rudinow 1994: 127, 130; Young 2005: 141). It has also been argued that cultural appropriation is sometimes profoundly offending, leading to the humiliation of some cultural group (Young 2005: 135). Sometimes the objection to cultural appropriation is simply that it undermines the autonomy or the right to self-identification of the group, even though the borrowing would not directly lead to the group's deprivation or ridicule (see Matthes 2016: 347). Interestingly, philosophers have been less concerned about cultural appropriation than scholars from other disciplines, maintaining that in many cases cultural appropriation is morally acceptable and arguing that often we should talk about "borrowing" rather than "appropriation." (Matthes 2016: 344; see also Lindner \& Nicklas 2012).

In this paper our aim is not to evaluate standard arguments against cultural appropriation, or the responses to those arguments. Instead, we consider what the moral doctrine called conventionalism says about cultural appropriation. According to conventionalism, an action is morally right if and only if it conforms to the conventions of society. In its pure form, conventionalism is in many ways a problematic doctrine, but we strive to show that moderate conventionalism does not face the problems of pure conventionalism, and that moderate conventionalism is a rather plausible view. According to moderate conventionalism, existing social conventions - understood as norms of "positive morality" that tell us what is acceptable and what is not - are morally relevant facts that should be taken into account when choosing how to act, whatever the content of the conventions happens to be. The claim is analogous with the traditional view that laws have some moral relevance and binding force independent of their content. Moderate conventionalism does not have strong implications for the debate concerning cultural appropriation, but it does have some. Our task here is to specify what those implications are.

\section{In Defense of Moderate Conventionalism}

Let us start with pure, rather than moderate, conventionalism. According to one version of (pure) conventionalism, an action is morally right if and only if it conforms to the conventions of society. To the extent that conventionalism has any intuitive plausibility, it derives from the fact that cultures and countries have 
somewhat different traditions and cultural habits, and in many cases it makes sense to follow the local standards rather than norms that are alien to most people involved.

It is important to note that (pure) conventionalism is not a form of moral relativism. According to moral relativism, no universal criterion exists for morally right actions. Conventionalism is based on the idea that there is a universal standard, namely the standard that an action should conform to local conventions. If the local conventions of politeness ask you to leave some food on the plate after dining, then you should leave it. But if the local conventions ask you to eat everything, then you ought to do so, given of course that the local conventions require politeness in the first place. Conventionalism resembles utilitarianism and many other moral doctrines that also have a universal standard of right action (an act is right if and only if it maximizes happiness) but require different actions in different circumstances.

Conventionalism is sometimes criticized by claiming that it runs into what is known as the reformer's dilemma (Feldman 1978: 166). The reformer's dilemma in this context is that conventionalism seems to imply that all reforms are always wrong. However, all reforms are certainly not always wrong, and therefore conventionalism is a problematic view, according to the critics. This objection is only partly convincing, since it is possible for one of the conventions to be that reforms should happen. If such a convention exists in society - as it often does then reforms seem to be consistent with conventionalism after all. The only problem that arises is the need to choose which convention to follow - and conventionalism cannot help in that decision.

A more serious problem with conventionalism is that it does not allow an independent moral evaluation of conventions. Surely this seems to be sufficient reason to doubt conventionalism. Conventions differ, and we can evaluate them not only by using other conventions but also by standards that are not conventions at all. We can rationally judge how good (or bad) a particular convention is. Conventionalism seems to deny this, making the doctrine highly implausible.

The doctrine we call moderate conventionalism offers a much more plausible view than pure conventionalism (cf. Velleman 2013). According to moderate conventionalism, social conventions are morally relevant and should be taken into account when deciding how to act. (The doctrine does not provide a criterion for morally right actions.) The claim here is not only that conventions are social facts that should be taken into account in decision-making, because all facts should be taken into account in decision-making (cf. Räikkä 2014: 10-11). That is, conventions are not seen only as "constraints" (that are sometimes easily removable and sometimes not so easily removable). Instead, according to moderate conventionalism, conventions have some "moral force" and they should be respected - if there is not sufficient reason not to respect them. Conventions are features that are morally relevant. In that respect they are like laws that might also carry moral weight, at least in democratic countries (see Wendel 2014: 89-105).

Why does moderate conventionalism have plausibility? There are many reasons. First, respecting existing conventions preserves social stability and societal cohesion. Although it is impossible to infer how things ought to be in a society from how they actually are, the current state of affairs can serve as a guide for behavior, helping to avoid causing too much disturbance. This avoidance of harm 
from social disruption makes it morally good to conform to the ways of society, at least to some extent. In the case of laws (that form an important part of conventions), we may have a general obligation to obey them, but this does not imply that we cannot at the same time resist many of them.

Second, it is usually morally wrong to act disrespectfully towards fellow citizens. It is important to respect the convictions and beliefs of other citizens. Conforming to the ways of society maintains a respectful distance from meddling with what people hold personally important or even sacred. The basis of liberal culture and society is the idea that people's opinions should be respected, because they are their opinions, not because of the content of those opinions. We could say that all opinions have an author, and to respect authors is to respect their views, at least to some degree. This is how we can value personal autonomy and people's capacity to determine their lives.

Third, we ought to respect the feelings of other people. Most of us have an emotional relationship to some social conventions, and we harm people by changing the conventions that are considered emotionally crucial. Thus, respecting conventions also protects the feelings of fellow citizens, which leads to overall positive consequences. Although argumentatively a mild notion, this avoidance of upsetting other people is what makes moderate conventionalism plausible. Notice that we can have an obligation to respect the emotions people have, whether or not we consider them particularly rational - whatever the rationality of emotions might mean.

Moderate conventionalism refers to morality, instead of merely "positive morality" (understood as existing moral norms) of a society. Moderate conventionalists hold that conforming to social conventions is a prima facie right for the reasons described above. Of course, if the social conventions are unfair or otherwise unacceptable, then it is permissible and even obligatory to criticize them. Societal conventions that rely on apartheid policies have moral relevance, since they are conventions adopted by some people, but obviously there are dozens of reasons not to follow those conventions, despite their prima facie moral force. Needless to say, although there are many reasons why conventions might have prima facie importance, they can always be overridden.

\section{Moderate Conventionalism and Cultural Appropriation}

Moderate conventionalism has some interesting implications for the debate on cultural appropriation. Consider the Jeans Argument:

P1. If outsiders' use of traditional Sami outfits in funny TV shows is morally problematic, then it is also morally problematic to use jeans (in funny TV shows), as they form a part of the group identity of the American working class.

P2. But obviously the use of jeans is not morally problematic in funny TV shows.

C. Therefore, outsiders' use of traditional Sami outfits is not morally problematic either.

The structure of the Jeans Argument is rather common in debates concerning different forms of cultural appropriation (or cultural borrowing), and the most 
common reply to the arguments that share this structure is to reject its first premise. For instance, in the case of the Jeans Argument, one can argue that using Sami outfits is morally problematic, since Sami people are a potentially vulnerable indigenous group, whereas the members of the American working class are relatively rich, and its group identity may be non-existent (although Bruce Springsteen would probably disagree). Moderate conventionalism provides a novel reason to reject the first premise of the arguments that share the structure of the Jeans Argument. In some cases, a morally relevant difference exists between the cases that are presented in the argument. This is the difference that only one of the practices is supported by the existing social conventions. Possibly, one of the differences between the use of the traditional Sami outfit in a TV show and the use of jeans in a TV show is that social conventions restrict the use of the Sami outfit, whereas they do not restrict the use of jeans. Social conventions may allow the use of jeans. This is a morally relevant difference, given that moderate conventionalism holds. (No doubt, it is often difficult to name the conventions, especially if some people deny their legitimacy. Note also that we may encounter a situation in which it is completely appropriate to use Sami outfits and where there is nothing insulting or unconventional in such behavior.)

The Jeans Argument and all the other arguments that share its structure are based on the idea that there are two cases that do not have morally relevant differences. But such a difference does exist, if one of the practices is prohibited (or supported) by the existing social conventions and the other is not - that is, if there is a convention difference (as there is, ex hypothesi). A convention difference can be said to exist when one case is prohibited by conventions and the other one is not. This difference is morally relevant, but the point here is not to claim that it is morally relevant solely because the other is prohibited and the other is not. There can be many moral reasons why the difference should be taken into account in decision-making.

Given that moderate conventionalism holds, those who would like to use arguments that resemble the Jeans Argument need to improve their reasoning when there is a convention difference. They should not claim that the two cases have no morally relevant difference. This claim would be false when a convention difference exists. Instead, they should either drop the argument, or show that, even though there is a morally relevant difference, the cases have relevant similarities too, and that these similarities are, morally speaking, more important than the difference related to social conventions. Perhaps this can be shown rather easily in some cases. But issues should be resolved on a case-by-case basis.

Our argument moves the discussion one step further. The crucial question, in many cases, is whether the (convention) difference between the cases is more or less important than the alleged similarity. The Jeans Argument, in all variations, is based on the observation that there is a morally relevant similarity between two (or more) cases. Here the representatives would say something about the "relative moral strength" of the relevant similarity.

Of course, whether a convention difference exists between the cases depends on the particular society and its conventions. For instance, the convention (or the conviction) that it is inappropriate to use the traditional Sami outfit in funny TV shows can be found in some societies, but not in others. Even within a single country it may be difficult to tell what, if any, the local convention is. When no 
clear convention exists on a matter, moderate conventionalism does not say much about it. Obviously, this shows that moderate conventionalism cannot play a major role in debates concerning cultural appropriation. But it is important to recognize that it can play some role.

The argument we have presented is clearly conservative in the sense that we refer to existing cultural conventions. We have a bias in favor of status quo, you might say. However, the implications of the conservative argument seem rather liberal. We consider the "conservatism" of our argument to be a merit rather than a burden, for the argument may - ideally - convince conservatives, and not only liberals, who already agree that in many cases cultural minority groups (such as Sami people) are not relevantly analogous to more powerful groups (such as the American working class). The argument presented above should be separated from the more general view that diversity itself is a good thing. The argument based on moderate conventionalism does not presume the value of diversity - unless there is a convention that supports its value (see also Young 2008; Strang \& Busse 2011; Brown \& Kopano 2014).

\section{Objections and Replies}

Before concluding, we would like to discuss some possible objections to the points we have made.

First, it can be argued that, in fact, no "social conventions" exist: it is fiction that there are historical and shared customs. According to this line of thinking, claims of "common practices" and "shared conventions" are false, and they are endorsed by those who would like to stay in power and define the alleged "essence" of their community so that it serves their own purposes. This argument, however, is implausible, at least when it is interpreted as a general claim about communities, societies and cultures. Surely we have conventions, although what they are is sometimes controversial and some people may try to steal the right to define them. Generally speaking, conventions do exist, although they are not stable and fixed over time. For instance, in many Western countries, it is inappropriate to use the word "fatso," and the fact that some people do use the word does not show that the convention does not exist. It only shows that some people do not want to conform to all conventions. (The term "convention" has various meanings. Some actions, such as how one dresses, are considered "conventional" when they are "usual." This is not what we have in mind here. Notice that conventions need not be very specific, but can take a rather general form such as "behave politely." Obviously, conventions may conflict with each other.)

Second, it can be objected that, although there are conventions in general, no specific conventions exist that are relevant in debates concerning cultural appropriation. This objection is based on the observation we already mentioned: even within a country it may sometimes be difficult to tell what the local convention is, and moderate conventionalism does not say much when there is no clear convention about a matter. The second objection is serious, but we are optimistic that in some cases there are relevant conventions that can possibly contribute to particular debates concerning cultural appropriation. How often relevant conventions exist is really an empirical question, and our aim is not to speculate on it here. However, it should be noted that not all conventions concern society as a whole. Conventions can vary within a society. When a group of people 
share a view concerning what is right or wrong, they share a convention. Different behavior is expected in churches, sporting events and government bureaus. Your conventions may not be our conventions. Multicultural societies contain a variety of seemingly conflicting conventions. Understanding the limits of one's own conventions may contribute to social cohesion as well.

Finally, it can be argued that moderate conventionalism does not play any role in the argument we have presented. Surely the idea that conventions should be respected if there are no specific reasons to oppose them can be defended, without relying on moderate conventionalism. Utilitarianism, for instance, encourages people to respect conventions, given that their overall consequentialist value is higher than the consequentialist value of their rejection. This objection is perhaps plausible, but we do not really care whether or not it is. If the prima facie moral importance of conventions can be shown by various moral doctrines, this sounds like good news for us. We have tried to argue that convention difference is a morally relevant difference - although this has gone unnoticed in the debate. If traditional moral doctrines support the view that convention difference is morally important, then the case we have made sounds rather plausible, we hope.

\section{Concluding Remarks}

We have argued that moderate conventionalism is not an implausible moral view. According to moderate conventionalism, existing cultural conventions and moral practices have prima facie moral force in the sense that they deserve some esteem, irrespective of whether they are morally ideal, since they are upheld by people. The view does not imply that conventions cannot be open for radical criticism. Obviously, in a good many cases, conventions need to be changed and their moral weight is rather weak, given their content. Still, it is important to notice that existing conventions can even have decisive moral weight. We have argued that those who are interested in cultural appropriation should take this into account. In particular, they should take this into account when arguing that two social phenomena are identical or morally analogous, although in fact the phenomena have a convention difference where only one convention is supported or endorsed by the existing conventions while the other is not.

\section{Acknowledgements}

We would like to thank Eerik Lagerspetz, Nadim Khoury, Kasper LippertRasmussen, Annamari Vitikainen, Anna-Karin Andersson, Susanne Uusitalo, Polaris Koi, Laura Puumala, Helena Siipi, Jari Kärkkäinen, Markku Oksanen, Jukka Varelius, Elisa Aaltola and the anonymous referees of Etikk $i$ praksis for their helpful comments on earlier versions of the paper. Mikko Puumala would like to thank Nessling Foundation for financial support.

\section{References}

Brown, T. L., Kopano B. N. (Ed.) (2014). Soul Thieves. New York, Palgrave Macmillan.

Feldman, F. (1978). Introductory Ethics. Englewood Cliffs, Prentice-Hall. 
Lindner, O., Nicklas, P. (2012). Adaptation and Cultural Appropriation. Oldenbourg: De Gruyter.

Lynskey, D. (2014, July 30). This means war: why the fashion headdress must be stopped. The Guardian. Retrieved August 20, 2018, from https://www.theguardian.com/fashion/2014/jul/30/why-the-fashionheaddress-must-be-stopped

Matthes, E. H. (2016). Cultural Appropriation without Cultural Essentialism? Social Theory and Practice, 42(2): 343-366.

Räikkä, J. (2014). Social Justice in Practice: Questions in Ethics and Political Philosophy. Heidelberg, Springer.

Rudinow, J. (1994). Race, Ethnicity, Expressive Authenticity: Can White People Sing the Blues? The Journal of Aesthetics and Art Criticism, 52(1): 127-137. http://doi.org/10.2307/431591

Strang, V., Busse M. (2011). Ownership and Appropriation. London, Bloomsbury.

Velleman, J. D. (2013). Foundation for Moral Relativism. Cambridge, Open Book Publishers.

Wendel, W. B. (2014). Ethics and Law. Cambridge, Cambridge University Press.

Young, J. O. (2005). Profound Offense and Cultural Appropriation. The Journal of Aesthetics and Art Criticism, 63(2): 135-146. https://www.jstor.org/stable/ 3700467

Young, J. O. (2008). Cultural Appropriation and the Arts. London, WileyBlackwell.

Young, J. O., Brunk, C. G. (Ed.) (2009), The Ethics of Cultural Appropriation, Chichester, Wiley-Blackwell.

Young, J. O. (1994). Should white men play the blues? Journal of Value Inquiry, 28(3): 415-424. https://doi.org/10.1007/BF01079504

Ziff, B. H., Rao, P. V. (Ed.) (1997), Borrowed Power: Essays on cultural appropriation, New Brunswick, Rutgers University Press. 